
\title{
Intelligent Spectrum Decision ML Algorithms for
} WSN

\author{
S. Sivaranjani*, S. Mohanraj and V. Kavitha \\ Department of ECE, M. Kumarasamy College of Engineering, Karur, Thalavapalayam - 639113, Tamil Nadu, India; \\ ssivaranjani.ece@mkce.ac.in, mohanrajs.ece@mkce.ac.in, kavithav.ece@mkce.ac.in
}

\begin{abstract}
Objective: To direct channel selection in WSNs. The tests indicate execution enhancements on the conveyance rate and conveyance defer when the proposed cognitive arrangements are utilized. Methods/Statistical Analysis: The utilization of administered Machine Learning (ML) for direct determination in WSNs. The proposed models were broke down utilizing ML apparatuses and strategies, and the best calculations were assessed on genuine sensor hubs. Findings: Wireless Sensor Networks (WSNs) utilize Industrial, Logical and Medical (ISM) range groups for correspondence, which are over-burden because of different innovations for example, WLANs and different WSNs. In this way, such systems must utilize astute strategies, for example, Cognitive Radio (CR) to exist together with different systems. The tests indicate execution enhancements on the delivery rate and delivery delay when the proposed psychological arrangements are utilized. Application/Improvements: Intelligent spectrum decision machine learning idea we can utilize all sort of use, for example, restorative field, Miltary applications, and so forth.
\end{abstract}

Keywords: Cognitive Radio, Delivery Delay, Delivery Rate, Machine Learning Algorithm, Wireless Sensor Networks

\section{Introduction}

An imperative issue in existing arrangements of wireless networks is the crowded nature of the electromagnetic medium ${ }^{1}$. Crowded range prompts a degraded network experience, requiring more retransmissions and in that capacity expanding the battery deplete of cell phones. Cognitive Radio (CR) is an innovation that handles this issue ${ }^{2}$. In the case of Wireless Sensor Networks (WSN), which operate on the ISM (Industrial, Scientific and Medical) band, CR functions improve the coexistence of multiple networks by ensuring a more efficient and reliable communication ${ }^{3}$. While the "Traditional" CRs select frequency bands or time intervals that are free of licensed users (the Primary Users), in WSN the CRs select channels with better conditions for communication, avoiding interference from WSN and other networks in the surroundings.

CR procedures for ISM endeavor to moderate the impedance among systems of various innovations. For instance, they can be utilized to designate a 802.15 .4 sys- tem to a channel having less cover with the encompassing 802.11 systems. Existing executions of ISM organizes as of now utilize intellectual radio systems: 802.11 APs working in the 5.2-5.7 GHz band utilize dynamic recurrence determination to decrease obstruction with radars ${ }^{4}$, and most APs change their channel task progressively. Further, remote systems can be sent in unforgiving situations. Electric apparatus and other hardware utilized as a part of modern plants may produce clamor that lessens the nature of correspondence ${ }^{5}$, so CR strategies might be utilized to adapt to this obstruction. Once the best channel is chosen, MAC conventions organize transmissions on that channel, lessening the parallel transmissions, for instance by planning transmissions amid sit still circumstances of different systems ${ }^{\underline{6}}$. This is known as range sharing, which is corresponding to channel choice.

This work proposes Machine Learning (ML) answers for channel determination (likewise referred to in the writing as range choice) in WSN. In spite of the fact that there are a couple of CR recommendations in the writing utilizing ML procedures in WSNs, those arrangements 
as a rule are not assessed on genuine hubs, and the vast majority of them are intended for gadgets with elite limit. This paper proposes and assesses in asset compelled equipment a ML display that considers the execution of past transmissions and gatherings, with a specific end goal to anticipate the level of control of each channel. Rather than assessing a solitary ML calculation, we examine the execution of numerous calculations, and additionally how each information parameter adds to the last yield of the model. At last, the most productive calculations were executed and tried on a proving ground. Results demonstrated that ML enhanced the execution of the WSN by decreasing the measure of transmission endeavors and the transmission delay in situations with obstruction from different systems.

As expressed in the past section, there are executions of CR answers for WLAN and different innovations. Despite the fact that they are exceedingly viable, the greater parts of them have high vitality utilization or computational many-sided quality, which blocks its utilization in WSN. Moreover, the appropriation of more strong equipment might be taken a toll restrictive, since most WSN arrangements require a high measure of sensor hubs. The proposition of CR for WSN utilize ascribes that are less demanding to gather, and require low computational and vitality assets. ML procedures are typically utilized in CRs to upgrade QoS measurements, or parameters got from at least one layers of the convention stack. One of the fundamental favorable circumstances in the utilization of ML systems is their capacity to display the conduct of the system in future interims, permitting the use of proactive arrangements, for example, channel exchanging $\stackrel{?}{ }$.

A standout amongst the most mainstream ML procedures in CRs is Artificial Neural Networks (ANN). A few creators propose the utilization of Self-Organizing Maps. The fundamental thought is to collect multidimensional examples, which are utilized to infer a grouped neural system, where the similitude of the neurons is controlled by the separation from each other. Each group speaks to a specific order. Notwithstanding, a few applications may request a substantial number of neurons keeping in mind the end goal to accomplish an adequate precision. This causes an exponential increment in the forecast time and memory utilization, henceforth rendering ANN too exorbitant to convey on some WSN stages. A couple of examinations use Partially Observable Markov Decision Processes ${ }^{\underline{8}}$, and Reinforcement Learning. The basic burden of these frameworks is the dependence of extremely correct reward capacities. Many WSNs, by having limited equipment, can't play out the figuring required for these capacities in practical time or with the important exactness. This work receives ML models that, even with the lessened accuracy of existing stages, give sufficient execution. Other than utilizing ML, numerous works in the writing utilize diverse ways to deal with display range choice in CRs. Crafted by reviews late deals ${ }^{2}$ with CRs. The creators overview channel determination and channel portrayal (considered by the creators as a past stage to channel choice), and additionally reconfiguration. The creators additionally characterize the best in class into unified and conveyed choice calculations.

The two range choice techniques for WSNs ${ }^{10}$. The first depends on RSSI (Received Signal Strength Indicator) and CCA (Clear Channel Assessment) estimations of each channel. The second technique depends on physical layer information and application necessities, and the importance of each information is a component of its entropy. Those strategies, be that as it may, were not assessed on genuine WSNs. Some CR answers for WSNs depend on vitality as it were. In two methodologies are tentatively examined: on the time space, where just a single channel is detected, and on the recurrence area, where various channels is detected, in this way understanding the positioning and choice of the best channel. Both methodologies utilize just the RSSI as credit to decide how occupied is the detected channel. Actualizing CR in genuine WSN has been an inexorably normal concern. In the creators analyzed three expectation strategies, deciding the possibility of its usage on a business WSN stage. The focal point of is not quite the same as our own: they evaluate the correspondence quality among neighboring hubs for directing, while in this work we gauge the nature of correspondence in an arrangement of channels, with a specific end goal to pick the best one.

This article is sorted out as takes after. The ML definition is displayed in Section 2, and the preparation set is exhibited in Section 3. Area 4 exhibits an exactness examination of the ML calculations, and an affectability test on the info parameters is talked about in Section 5. The assessment on a genuine stage is portrayed in Section 6. A discourse of the vitality utilization of the proposed arrangements is exhibited in Section VII. Section VIII reaches the determinations and future work. 


\section{Spectrum Decision Employing ML}

This section reveals our channel indicator, which was displayed utilizing ML. The indicator is the focal bit of our range choice approach, since it assesses the nature of each channel. Having a gauge of the connection quality, the range choice errand is decreased to picking the channel with the best quality. We at first built up an order based indicator, partitioning channel into classes of channel quality from best to most noticeably awful. Notwithstanding, after preparatory tests, we watched that the aftereffects of class partition were poor. Utilizing degeneration based indicator, which yields a genuine esteem, demonstrated more powerful.

In this way, in this paper we propose a degeneration based indicator, which approximates the nature of a connection by assessing the quantity of transmission endeavors for the following $\mathrm{N}$ parcels (the expectation window), where $\mathrm{N}$ is a configurable esteem. This yield was picked on the grounds that the quantity of retransmissions indicates the nature of the connection, since it is impacted by external and inner obstruction. A channel that transmits information with less endeavors is a channel with better execution, since it will display bring down postponements and misfortune rates. Figure 1 demonstrates a disentangled rendition of the indicator. With a specific end goal to create the expectation, we should gather various data sources (set 1 in the past condition). The following attributes were selected as input for our predictor:

- Received Signal Strength Indicator (RSSI): Quantifies the received signal strength on the radio. A fixed number of samples are collected before each transmission. In our work, this metric is read from the transceiver hardware.

- Number of Transmission Attempts: Number of attempts made to transmit a packet, successfully or not, until either the packet is successfully sent or the node gives up.

- Reasons of each Failed Attempt: The cause of retransmissions are also accounted for, as follows:

- ERX: The radio was busy receiving another packet.

- ECCA: CCA algorithm shows that the channel is busy.

- ENACK: The radio transmitted a packet but its acknowledgment has not been received.
- Performance Data from the Last Received Packet: The RSSI and the LQI (Link Quality Indicator) from every received packet are collected.

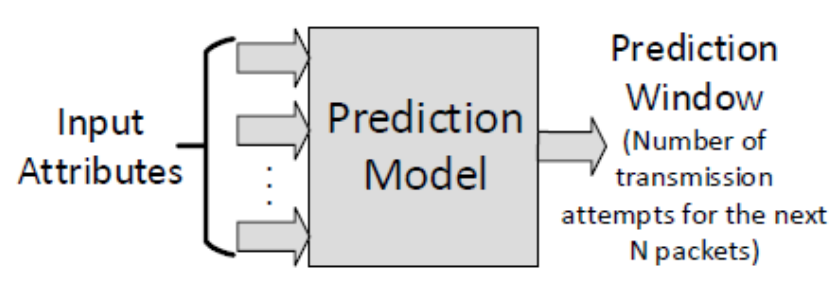

Figure 1. Regression-based predictor.

As the input values vary significantly at each transmission or reception, they are sampled many times and aggregated. This summarizes the recent history of the past transmissions and also filters outliers and spikes. A weighted moving average was used for this purpose. Equation (1) shows the calculation of the moving average, where $I_{t}$ is the input value at time $t$, and $U_{t-1}$ is the moving average value at time $t-1$. The value of $\infty$ determines the smoothness and responsiveness of the signal. In order to prevent a decision based on unreliable data, the medium should be probed several times. We call this sequence of probes the sensing window of the predictor. Smaller values of $\alpha$ generates better predictors, as will be evaluated in Section IV, however, it demands more data, since the variation between samples is small, and the convergence time will be longer.

$$
U_{t}=U_{t-1}(1-\infty)+I_{t} \alpha
$$

The most complex and costly part of Machine Learning based algorithms is its training. In our algorithm, training occurs only once, before the network is deployed. Only a few sensor nodes are required to gather the input data, while the processing occurs on a PC. The final code deployed on the sensor nodes contains only the final Machine Learning model. In order to achieve best results, the training process could be repeated before each deployment, capturing the characteristics of the medium on each location. However, if the training set is diverse (that is, having many types of sources interference and types of network coexisting), a single training set could be used for many deployments. 


\section{Data Collection for the Training Bases}

We captured data for the training sets using scenarios with varying degrees of interference and coexistence. The scenarios aim to obtain a generic training set, so that the prediction models are independent of the topology and types of coexisting networks. We collected data from three different scenarios:

- Low Interference: Only the sensor nodes were transmitting data among themselves, with low Wi-Fi interference.

- Average Interference: In addition to the data transmitted by the WSN nodes, two Wi-Fi routers transmitted data among themselves in low power ( $5 \mathrm{dBm}$ ), and distant from each other (six meters), in the same frequency range used by the WSN.

- High Interference: Similar to the Average Interference scenario, however the routers were close to each other and the WSN (approximately thirty centimeters), and transmitting at a higher power $(20 \mathrm{dBm})$.

We selected the Iris WSN platform ${ }^{11}$ as the collection platform. The WSN consisted of ten sensor nodes, each connected to a computer for performance data storage. Each node transmitted 28-byte packets every 500ms. Before each transmission, ten RSSI samples were collected, which makes up the list of selected attributes, presented in Section II, and at the end of the transmission the remaining attributes were also collected. The sensor nodes were placed in pairs, so that the effects of randomness in the performance of ML algorithms could be reduced.

\section{Accuracy Analysis of the ML Model}

Using the training set from the previous section, we used Matlab to measure the accuracy of the proposed ML model using different ML algorithms. All the algorithms that generated a model within a reasonable time (less than one week) were considered in this work. For this reason, SVM (Support Vector Machine), considered the state of the art in ML, was discarded. The algorithms evaluated are listed below:

- Function-based algorithms: Isotonic Regression, Linear Regression, MLP Regression, Multilayer Perceptron, Pace Regression, RBF Regression.

- Search-based algorithms: K-Nearest Neighbors $(\mathrm{KNN})$
- Algorithms based on meta-learning: Additive Regression with Decision Stump as base algorithm, Bagging - with REP Tree as base algorithm.

- Algorithms based on decision rules: Conjunctive Rule, Decision Table, M5 Rules.

- Algorithms based on decision trees: Decision Stump, Extra Tree, M5P, REP Tree.

We used the Ten-Fold Cross Validation method, thereby obtaining one hundred training-test combinations for each algorithm. In order to rank the algorithms, we used the paired t-test, which considers the dependency among samples, and therefore is the most suitable for use in conjunction with Ten-Fold Cross Validation. We adopted the Root Mean Squared Error (RMSE) as the main performance metric which is more resilient to outliers, an important factor in WSNs due to sharp fluctuations of the electromagnetic environment. The performance analysis of each algorithm was divided into two stages. The first one analyzed the impact of $\alpha$ on the performance of the ML algorithms. The second stage analyzed the influence of the size of the prediction window on the performance of each ML algorithm.

\subsection{Evaluation of $\alpha$}

We measured the RMSE for values of $\alpha$ between 0.01 and 0.09 with a step of 0.02 . For each value of $\alpha$, a distinct training set was created. The size of the prediction window was fixed at 10 , of which the sum of transmission attempts was scaled into a range between 0 and 100 . Table 1 presents the average RMSE obtained in ten runs of each $\mathrm{ML}$ algorithm. We observed that a reduction in the value of acauses an increase in the performance. This is due to the fact that, the smaller the value of $\alpha$, the lower the variability of the input values. As we have considered three different scenarios, the low variability in the input values causes the training set to approach three distinct clusters, each of them related to a collection scenario. This in turn reduces the complexity of the models generated by the regression algorithms, and thus increasing their performance.

\subsection{Evaluating the Size of the Prediction Window}

The length of the expectation window impacts the quantity of transmission endeavors to be assessed, in this manner affecting the accuracy of the indicator: it ought to be simpler to foresee the performance of more trans- 
Table 1. Root mean squared error: varying A.

\begin{tabular}{|l|l|l|l|l|l|}
\hline \multirow{2}{*}{ ML Algorithm } & \multicolumn{5}{l}{ Weight $(\boldsymbol{\alpha})$} \\
\cline { 2 - 6 } & $\mathbf{0 . 0 1}$ & $\mathbf{0 . 0 3}$ & $\mathbf{0 . 0 5}$ & $\mathbf{0 . 0 7}$ & $\mathbf{0 . 0 9}$ \\
\hline Isotonic Regression & 8.32 & 8.26 & 8.29 & 8.32 & 8.34 \\
\hline Linear Regression & 8.50 & 8.54 & 8.82 & 9.12 & 9.43 \\
\hline MLP Regression & 7.99 & 8.00 & 8.03 & 8.07 & 8.10 \\
\hline $\begin{array}{l}\text { Multilayer } \\
\text { Perceptron }\end{array}$ & 8.68 & 8.77 & 8.78 & 8.83 & 8.80 \\
\hline Pace Regression & 8.50 & 8.54 & 8.80 & 9.10 & 9.39 \\
\hline RBF Regression & 8.20 & 8.09 & 8.11 & 8.17 & 8.23 \\
\hline KNN & 3.64 & 3.75 & 4.06 & 4.98 & 6.30 \\
\hline Additive Regression & 8.06 & 8.06 & 8.06 & 8.07 & 8.10 \\
\hline Bagging - REP Tree & 6.29 & 7.00 & 7.34 & 7.55 & 7.67 \\
\hline Conjunctive Rule & 8.83 & 8.80 & 8.80 & 8.79 & 8.79 \\
\hline Decision Table & 7.74 & 7.91 & 7.98 & 8.02 & 8.03 \\
\hline M5 Rules & 8.32 & 8.52 & 8.53 & 8.03 & 7.98 \\
\hline Decision Stump & 8.91 & 8.84 & 8.82 & 8.80 & 8.82 \\
\hline Extra Tree & 6.40 & 7.90 & 8.84 & 9.47 & 9.90 \\
\hline M5P & 7.07 & 7.74 & 7.73 & 7.86 & 7.93 \\
\hline REP Tree & 6.78 & 7.58 & 7.86 & 7.98 & 8.03 \\
\hline
\end{tabular}

missions, since the impacts of commotion and mistake would be littler on the general yield. As in the past stage, for every window measure that was tried, a relating preparing set was made, with a similar scaling of the forecast window utilized as a part of the past area. In these tests, the measure of the forecast window was changed in the vicinity of 1 and 30, with a fixed at 0.01 , which demonstrated the best execution in the past area. Figure 2 displays the normal RMSE acquired in ten keeps running for the five ML calculations with the best execution on the previous assessment. We dispose of the aftereffects of the other algorithms for comprehensibility reasons, however the patterns are like those demonstrated here. We watch a logarithmic conduct for all the algorithms, while expanding the measure of the forecast window. This shows bigger windows are prudent, however the advantages decrease as the window measure increments.

\section{Sensitivity Analysis}

The objective of affectability examination in ML is to survey the commitment of each info credit to the yield of the indicator. As such, it evaluates the significance of

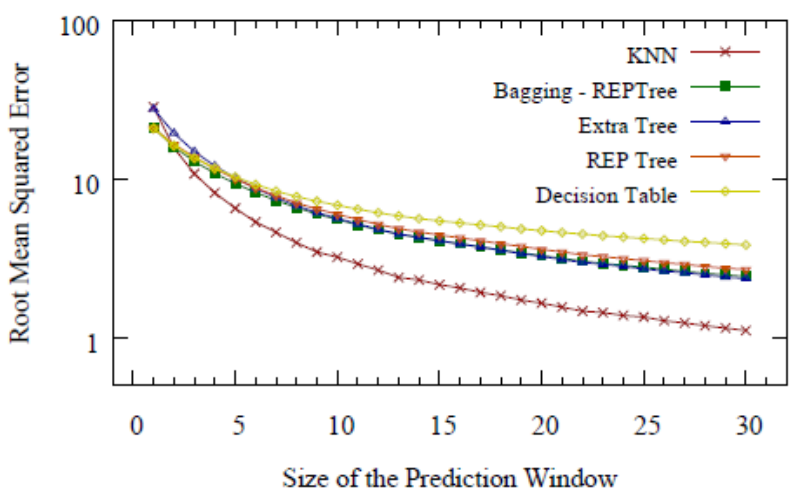

Figure 2. Root Mean Squared Error - Varying the size of the prediction window.

each gathered data on the channel quality estimation. This data is helpful, if there is a need to diminish the measure of information qualities, for instance if the model is excessively mind boggling, making it impossible to be instantiated on genuine WSNs, so characteristics that reason next to zero effect are expelled. Further, affectability investigation additionally recognizes properties that intensify the forecast. With a specific end goal to acquire the effect of each trait on the forecasts of the ML calculations, a progression of preparing sets have been made.

For every one, we evacuated one of the traits, and re-inferred the model. At that point, we thought about the RMSE of the entire model against the RMSE of the diminished models. It was watched that the expulsion of a few traits produced little upgrades (under 10\%) in the RMSE. Those enhancements happened with the evacuation of the RSSI traits gathered before every transmission, and in addition the LQI and the RSSI gathered on every gathering. One explanation behind the change with the evacuation of RSSI would be the low determination of the gathered esteems, because of equipment restrictions. We additionally watched the most astounding drop in the RMSE for ECCA and ENACK, showing that those parameters are the most vital in the model, and thusly ought to get more consideration in future work. For instance, we could devise a more refined preprocessing calculation for those information sources.

\section{Hardware Implementation}

To assess how the ML models admission, all things considered, we actualized them over a basic channel determination convention. This area demonstrates exploratory 
outcomes generally advantageous and most noticeably bad situations, with low and abnormal amounts of impedance, individually. The hubs were arranged to work in five back to back channels, however more channels could be effortlessly bolstered. Six sensors were utilized, reenacting a bunch of the sensor arrange. The Central Node, a hub picked discretionarily in the group, executes the proposed range choice model. Alternate hubs, called Helper Nodes, were settled on every one of the five assessed channels. The reason for these hubs is to speak with the Central Node when required. In spite of the fact that for this assessment we utilize one Helper Node for each channel, the convention can be altered so just a single Helper Node can be utilized to detect all channels alongside the Central Node. The strategy for the decision of a Helper Node, and also the decision of the Central Nodes (considering a WSN formed by hundreds or thousands of hubs), is left as future work.

Four ML models were assessed. The chose ones were the best performing calculations of Section V that likewise fit the memory and handling necessities of the Iris stage. This stage was picked in light of the fact that it has extremely unobtrusive equipment, and if the proposed arrangements chip away at Iris they in all probability will deal with all the more effective stages. One model of each class introduced in Section IV was executed: Rep Tree, M5 Rules, Additive relapse with Decision Stump as base calculation, and Isotonic Regression. As gauge, two arrangements were actualized. The to start with, called Fixed, works on a settled channel and does not perform range detecting. The second, called RSSI, was propelled by ${ }^{12}$. It gathers 100 RSSI tests in each detected channel, and chooses the channel with the littlest entirety of RSSI tests. Table 2 demonstrates the ROM and RAM memory utilization, in bytes, for the actualized models. All calculations were executed utilizing drifting point factors, which are imitated in programming on the Iris stage since the chip does not have a scanning point unit. A more proficient usage in view of whole numbers is outside the extent of this work.

As expected, the Fixed solution showed the lowest memory consumption, since it has no prediction model implemented. Moreover, the M5 Rules solution showed the highest consumption, since it is based on a decision tree that requires an extensive amount of floating point numbers. The other solutions had similar memory consumption, mainly because those work with a similar amount of floating point values. The power consumption
Table 2. Memory consumption of the implemetations

\begin{tabular}{|c|c|c|}
\hline \multirow{2}{*}{ Prediction Model } & \multicolumn{2}{|c|}{ Memory Consumption(Bytes) } \\
\hline & $R O M$ & $R A M$ \\
\hline M5 Rules & 46492 & 1284 \\
\hline REP Tree & 24638 & 1284 \\
\hline Additive Regression & 24308 & 1284 \\
\hline Isotonic Regression & 24306 & 1284 \\
\hline RSSI & 22812 & 1363 \\
\hline Fixed & 21950 & 1271 \\
\hline Total Capacity & $120 \mathrm{~K}$ & $8 \mathrm{~K}$ \\
\hline
\end{tabular}

was not measured in this work, since its experimental measurement requires the execution of the algorithms for longer times (weeks of months), or the use of very sensitive multi-meters. The precise measurement of energy consumption $^{13}$ is thus left for future work, however a qualitative discussion is presented in Section 7.

\subsection{Low External Interference Scenario}

This scenario evaluates the WSN in an environment with low interference from other networks. Here, the Helper Nodes were positioned approximately six meters away from the Central Node, and the main source of interference was the WLANs in the vicinity of the test environment. Spectrum decision solutions and the baselines were run in sequence during 15 minutes each, and five independent experiments were performed. The results are presented with a confidence interval of $95 \%$. Table 3 shows the results.

Table 3. Results for the low interference scenario

\begin{tabular}{|c|c|c|c|c|}
\hline $\begin{array}{c}\text { Implemented } \\
\text { Solution }\end{array}$ & $\begin{array}{c}\text { Delivery } \\
\text { Rate (\%) }\end{array}$ & $\begin{array}{c}\text { Attempts/ } \\
\text { Packet }\end{array}$ & $\begin{array}{c}\text { Collisions/ } \\
\text { Packet }\end{array}$ & $\begin{array}{c}\text { Latency/ } \\
\text { Packet(ms) }\end{array}$ \\
\hline REP Tree & 96.67 & 1.79 & 0.39 & 6.91 \\
\hline M5 Rules & 97.23 & 1.73 & 0.24 & 6.56 \\
\hline $\begin{array}{c}\text { Additive } \\
\text { Regression }\end{array}$ & 98.75 & 1.34 & 0.17 & 5.84 \\
\hline $\begin{array}{c}\text { Isotonic } \\
\text { Regression }\end{array}$ & 92.89 & 1.96 & 0.51 & 7.34 \\
\hline RSSI & 98.00 & 1.63 & 0.25 & 6.44 \\
\hline Fixed & 91.99 & 2.52 & 0.64 & 8.71 \\
\hline
\end{tabular}

The proposed solutions were able to detect variations in the behavior of local WLANs, resulting in the choice of different channels and improving the performance of the 
links over the Fixed solution. However, the performance improvements vary for each algorithm. Only one of the spectrum decision solutions (Additive Regression) was better than the Fixed solution for all the analyzed metrics. For the other spectrum decision solutions, with the exception of Isotonic Regression, we could see an improvement on the average number of transmission attempts per packet. In addition to the average transmission attempts, M5Rules was also better than the same baseline according to the average latency per packet. The performance of the ML solutions, when compared to the RSSI solution, was very similar. The main source of interference was the signal generated by the WLANs, and was not significant as shown by the very high delivery rates even for the fixed channel approach. The spectrum decision solutions were built to handle sources of interference of greater magnitude, due to the characteristics of the training set. Thus, in cases where the interference is very low, those algorithms do not outperform the simple RSSI solution, although they provide a comparable performance.

\subsection{High External Interference Scenario}

In this section we evaluate the behavior of the proposed spectrum decision solutions in a coexistence scenario with heavy interference. The Helper Nodes were placed near the Central Node, at a distance of about thirty centimeters. As the main source of interference, in addition to the WLANs located in the test area, we placed two routers near the WSN transmitting data to each other, at high power and the maximum traffic possible with iperf. The WLAN was operating at frequencies next to those used by the WSN. In order to make the RF medium even more unpredictable, the routers communicate for 15 seconds, and then switch to another random channel. Due to timing limitations, the routers needed to pause for 10 seconds before each 15 second transmission burst, in order to guarantee that the channel change was successful. Table 4 shows the results obtained for five independent experiments and confidence interval of $95 \%$.

In this test scenario we observed significant improvements in the performance of the WSN, when comparing the proposed solutions against the baselines. This is due to the use of a wider range of attributes, which makes a difference when interference (internal or external) of greater magnitude is observed. In such cases, the use of RSSI readings may lead to bad choices, since the signalto-noise and interference ratio varies considerably. Thus,
Table 4. Results for the high interference scenario

\begin{tabular}{|c|c|c|c|c|}
\hline $\begin{array}{c}\text { Implemented } \\
\text { Solution }\end{array}$ & $\begin{array}{c}\text { Delivery } \\
\text { Rate(\%) }\end{array}$ & $\begin{array}{c}\text { Attempts/ } \\
\text { Packet }\end{array}$ & $\begin{array}{c}\text { Collisions/ } \\
\text { Packet }\end{array}$ & $\begin{array}{c}\text { Latency/ } \\
\text { Packet(ms) }\end{array}$ \\
\hline REP Tree & 94.73 & 2.63 & 0.32 & 8.02 \\
\hline M5 Rules & 93.86 & 3.22 & 0.34 & 8.34 \\
\hline $\begin{array}{c}\text { Additive } \\
\text { Regression }\end{array}$ & 93.36 & 2.98 & 0.40 & 8.93 \\
\hline $\begin{array}{c}\text { Isotonic } \\
\text { Regression }\end{array}$ & 95.71 & 2.31 & 0.24 & 7.86 \\
\hline RSSI & 88.97 & 3.37 & 0.42 & 9.56 \\
\hline Fixed & 88.12 & 3.56 & 0.43 & 9.98 \\
\hline
\end{tabular}

it is necessary to employ more sophisticated decision algorithms, such as those proposed in this work. Table 4 also suggests that the performance of the ML approaches is very similar, when analyzing the confidence intervals. Despite the differences in performance obtained in Matlab, the inaccuracies inherent of real deployments and the natural variations in the wireless medium may explain the similar performance on a real WSN. This also indicates that the predominant factor in the performance of a spectrum decision solution based on ML is the model and the selection of the input data, and not the choice of the ML algorithm. Therefore, we believe that, for spectrum decision in WSN, it is better to select the ML algorithm which generated the simplest prediction model in terms of computational resources.

\section{Discussion- Energy Consumption}

As discussed about in Section 6, the proposed ML models enhanced the system execution for all the deliberate measurements. Be that as it may, one can scrutinize the vitality utilization of the proposed convention, contrasted with the acquired advantages ${ }^{14}$. The detecting period of the convention will have high vitality utilization, in light of the fact that the sensor hub needs to send numerous tests and afterward process the information utilizing the ML models. The trials utilized a little transmission period, fading the vitality advantages of channel determination. The explanation behind a little interim was to get brings about a matter of weeks, since numerous trials were keep running for every design. In any case, in genuine organizations, the interim among detecting cycles can be higher than the one received in Section 6. The proposed ML mod- 
els were worked to choose the best channel utilizing an expansive expectation window, so they should keep a reasonable execution even with longer detecting interims ${ }^{15}$. With a bigger interim between detecting cycles, a decline in the vitality utilization is normal, which can conquer conventional MAC and impedance alleviation techniques that point on vitality utilization. The correlation with these strategies is left as future work. At long last, since most parameters of the models were picked observationally, a further report should be possible keeping in mind the end goal to enhance these parameters and decrease the detecting time of the convention, diminishing considerably more the vitality utilization. This examination is likewise left as future work.

\section{Conclusions}

Given the pollution of the ISM band and the power limitations in WSN, this article proposed the utilization of expectation models produced by ML calculations for the choice of the best working channel. Relapse models of low computational cost were manufactured in light of information gathered from genuine stages. These models gauge the execution of transmissions in the short and long terms, permitting the decision of the channel with better correspondence quality. The fundamental test in WSN is the memory and handling limitations. In this way, the proposed models were assessed in the Iris stage. The tests demonstrated that the utilization of ML builds the execution of the WSN when contrasted with settled channel and vitality based channel determination techniques. The fundamental execution picks up were found in conjunction situations with swarmed systems. As future work, we will add more information parameters to the model. Further, we will refine the pre-handling of the most vital info parameters. We will likewise endeavor to diminish the quantity of detected channels at every cooperation in view of past channel gauges. Besides, we will explore in the case of learning is doable by utilizing semi-regulated ML calculations.

\section{References}

1. Akyildiz IF, Lee W-Y, Vuran MC, Mohanty S. Next generation/dynamic spectrum access/cognitive radio wireless networks: A survey. Elsevier Computer Networks. 2006 Sep; 13(50):2127-59. Crossref.
2. Yucek T, Arslan H. A survey of spectrum sensing algorithms for cognitive radio applications. IEEE Communications and Surveys Tutorials. 2009; 1(11):116-30. Crossref.

3. Sherman M, Mody A, Martinez R, Rodriguez C, Reddy R. IEEE standards supporting cognitive radio and networks, dynamic spectrum access, and coexistence. IEEE Communications Magazine. 2008; 7(46):72-9. Crossref.

4. Tran T-D, Silva R, Nunes D, Silva J. Characteristics of channels of IEEE 802.15.4 compliant sensor networks. Wireless Personal Communications. 2012 Dec; 3(67):541-56. Crossref.

5. Glaropoulos I, Lagana M, Fodor V, Petrioli C. Energy efficient COGnitive-MAC for sensor networks under WLAN co-existence. IEEE Transactions on Wireless Communications. 2015 Jul; 7(14):4075-89. Crossref.

6. Bantouna A, Stavroulaki V, Kritikou Y, Tsagkaris K, Demestichas P, Moessner K. An overview of learning mechanisms for cognitive systems. EURASIP Journal on Wireless Communications and Networking. 2012 Jan; 1:22. Crossref.

7. Tsagkaris K, Bantouna A, Demestichas P. Self-organizing maps for advanced learning in cognitive radio systems. Computers and Electrical Engineering. 2012 Jul; 4(38):862-81. Crossref.

8. Tanwongvarl C, Chantaraskul S, Wehrle K, Spaniol O. Intelligent channel assignment in cognitive wireless sensor networks: Learning from virtual channel environment. Proceedings of International Chinese Information Systems Association; 2014. p. 1-4.

9. Kaarthik K, Sivagurunathan TP, Sivaranjani S. A review on spectrum sensing methods for cognitive radio networks. Journal of Advances in Chemistry. 2016 Nov; 8:5053-7.

10. Robert C, Moy C, Wang C-X. Reinforcement learning approaches and evaluation criteria for opportunistic spectrum access. Proceedings of International Cricket Council; India. 2014. p. 1508-13. Crossref.

11. Masonta M, Mzyece M, Ntlatlapa N. Spectrum decision in cognitive radio networks: A survey. IEEE Communications Surveys and Tutorials. 2012 Nov; 3(15):1088-107.

12. Correia LHA, Oliveira EE, Macedo DF, Moura P, Loureiro AAF, Silva JS. A framework for cognitive radio wirelesssensor networks. Proceedings of International Sustainability and Carbon Certification (ISCC); India. 2012. p. 611-6.

13. Silva VF, Macedo DF, Leoni JL. Machine Learning-based spectrum decision algorithms for wireless sensor networks. Proceedings of IEEE; Central North Carolina. USA. 2016. Crossref.

14. Stabellini L, Javed M. Experimental comparison of dynamic spectrum access techniques for wireless sensor networks. Proceedings of VTC-Spring; India. 2010. p. 1-5. Crossref.

15. Liu T, Cerpa AE. Data-driven link quality prediction using link features. ACM Transactions of Sensor Networks, 2014 Jan; 2(10):1-37. Crossref. 